\title{
ADAMANTO-ODONTOMA
}

\section{Report of CASE}

John W. Kemper, D.D.S., M.D.,* and R. W. Root, A.B., D.D.S.†

F W. (No. 552359), a white girl 6 years of age, was admitted to the Oral E. Surgery Department of the University Hospital with a chief complaint of swelling of the right side of the face. It was first noticed by her mother nine months previous to admission to the hospital. There was a gradual increase in size for about four months, after which the mother stated it seemed to decrease in size. During the past three months there had been no apparent change in size. There had been no symptoms other than the swelling.

The family, birth, and developmental histories were negative. There had been no previous diseases.

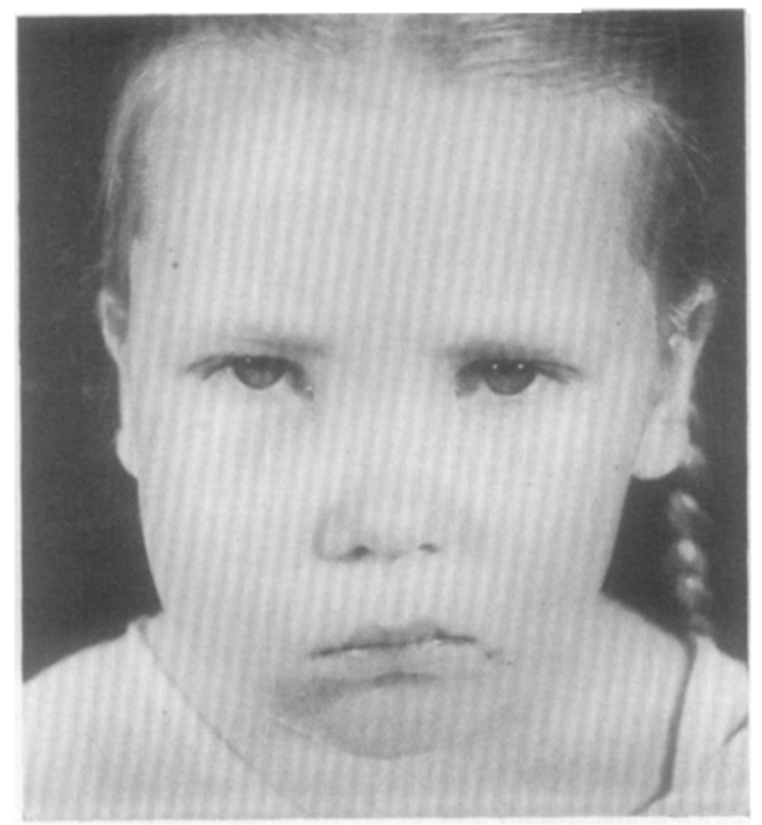

Fig. 1.-Photograph showing facial asymmetry caused by maxillary adamanto-odontoma.

Physical examination revealed a well-developed, well-nourished child with an obvious swelling on the right side of the face. The tumescence extended from inferior border of the orbit to the mid-portion of the check (Fig. 1). It was noninflammatory, stony hard and painless. It seemingly involved the whole right maxilla. Intraoral examination showed a hard, noninflammatory tumor of the maxilla extending from cuspid region posteriorly beyond the

From the Department of Oral Surgery, University Hospital, University of Michigan.

* Professor of Oral Surgery and Head of Department of Oral Surgery, School of Dentistry and University Hospital, University of Michigan.

tIntern, Department of Oral Surgery, University Hospital, University of Michigan. 


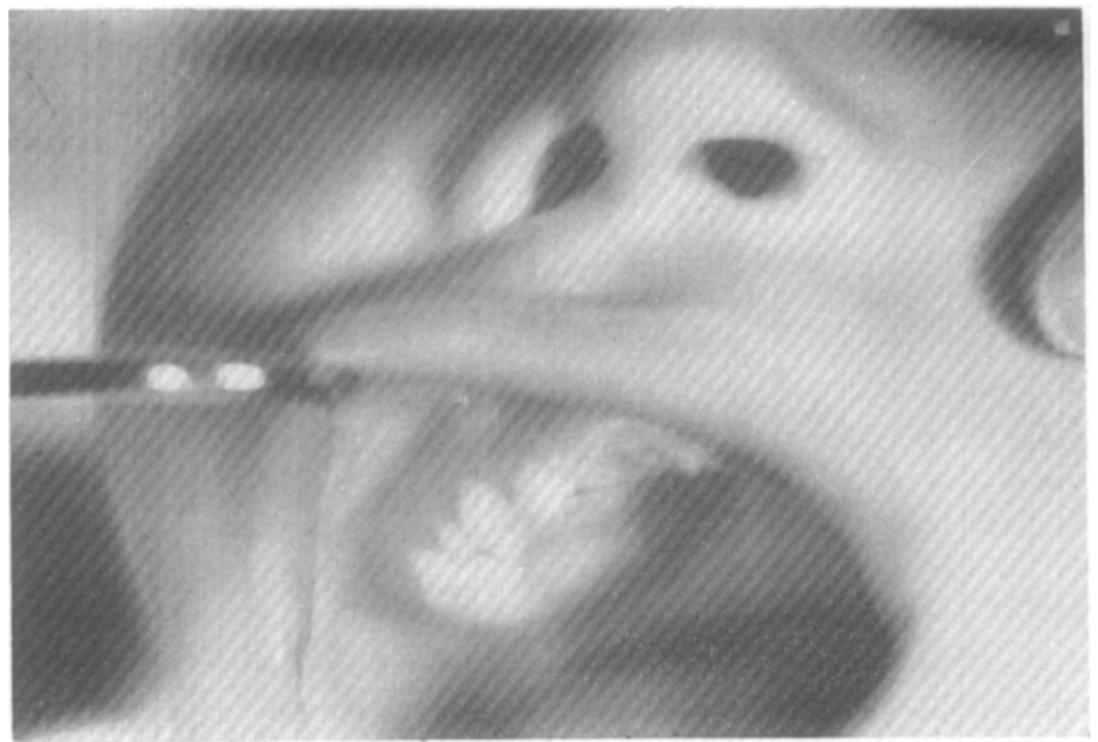

Fig. 2.--Intraoral photograph of maxillary adamanto-odontoma.

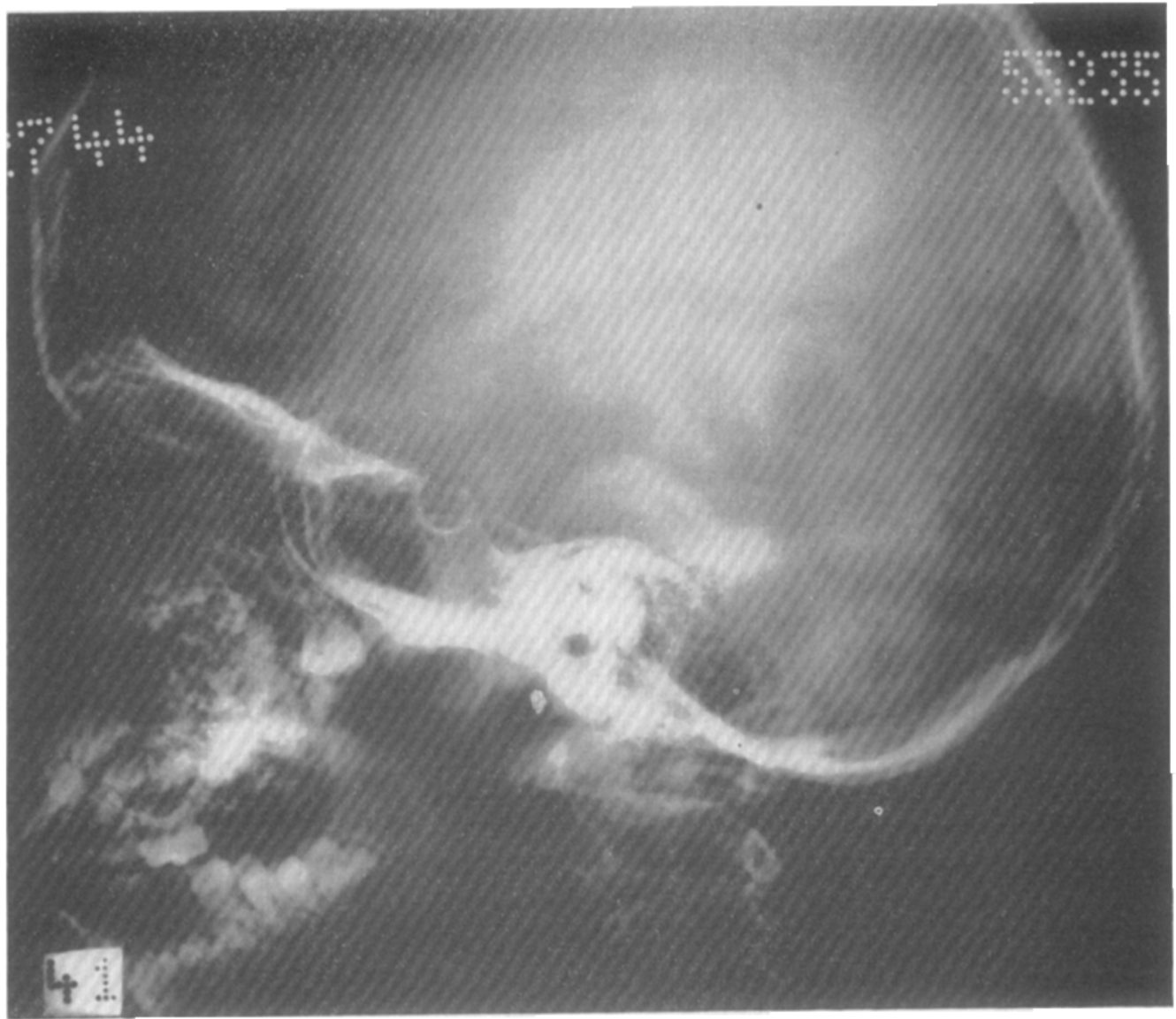

Fig. 3.-Lateral view' radiograph showing large maxillary tumor containing calcifed structures and the crowns of unerupted premolars and first and second molars. 
tuberosity. The expanded buccal plate of bone completely obliterated the canine fossa and upper mucobuccal fornix. There was bulging of the palatal process extending to the midline of the palate. The two maxillary incisors were missing and the right second deciduous molar was very mobile. The mass was bony hard in its entirety and was painless, with no evidence of suppuration (Fig. 2).

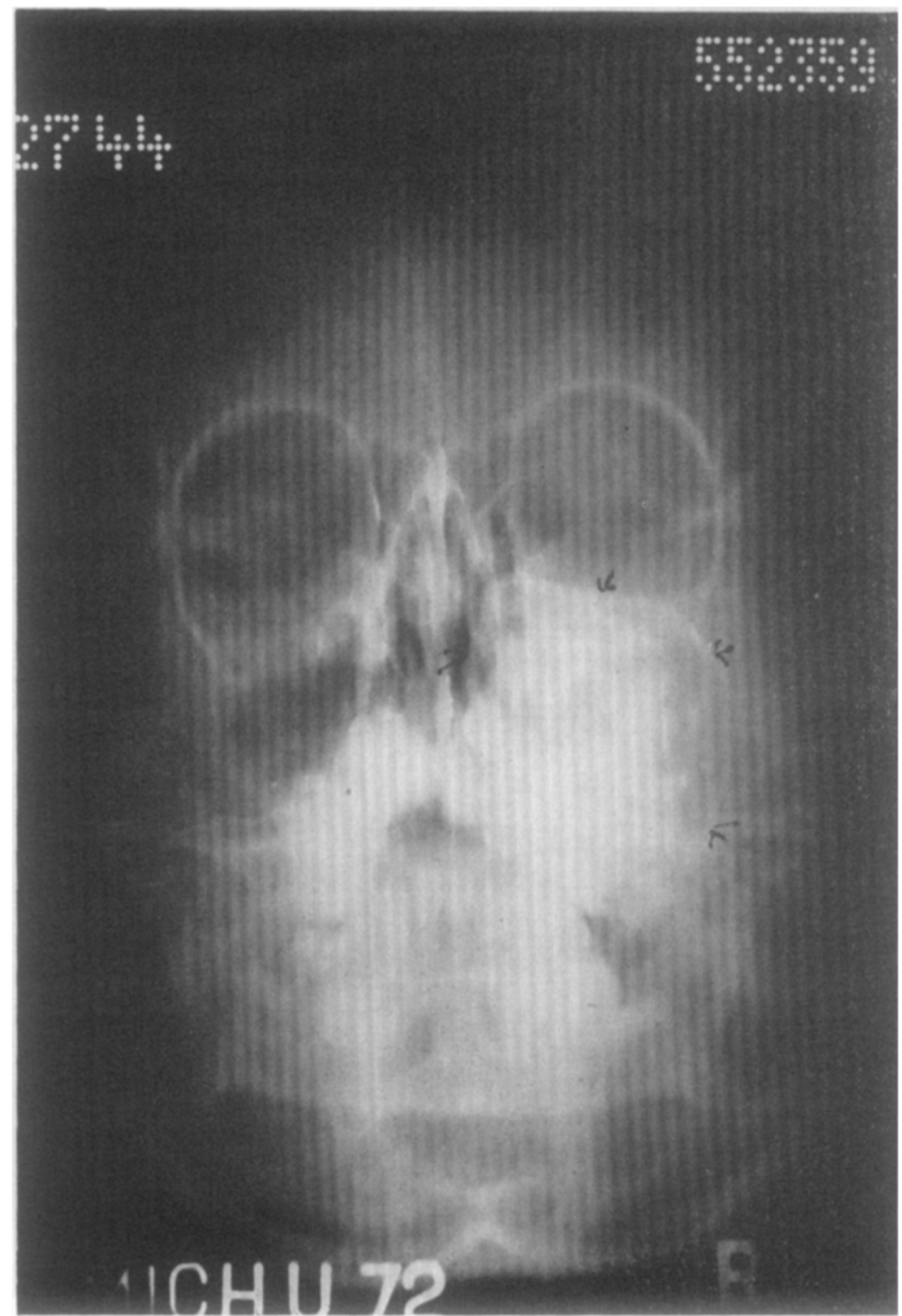

Fig. 4.-Posteroanterior radiograph showing relative size of the timor and expanded superior and lateral walls of the antrum.

The remainder of the physical examination was entirely negative.

Blood examination showed the hemoglobin to be 84 per cent and the white count 6,300 . The Kahn test was negative. The urine was negative, except 


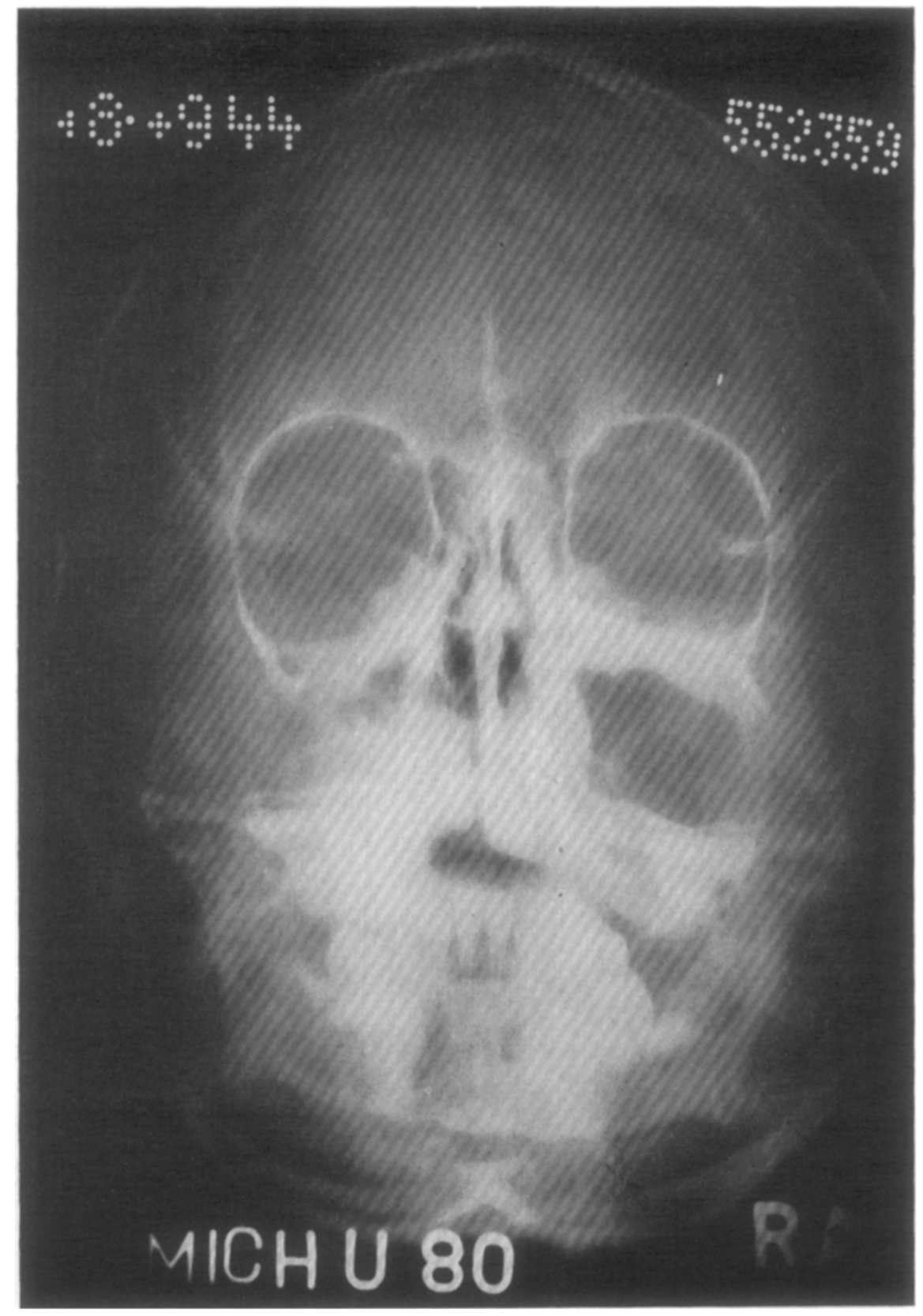

Figs. 5 and 6.-Same views as Figs. 3 and 4 after enucleation of the tumor. 
for 8 W.B.C. per high-power field. The tuberculin test was negative as was the admission chest film.

$X$-ray examination of the skull revealed a large tumor filling and expanding the right maxillary antrum to considerable proportions. The growth showed extensive patchy calcification with several crowns, perhaps of the second dentition, in the premolar and molar regions (Figs. 3 and 4 ). There was no evidence of invasion of the ballooned-out orbital, medial, and lateral walls.

Since there was so much expansion of the superior and lateral walls of the antrum without any apparent erosion, it led to the impression that this was most likely a benign lesion. This, together with the contained tooth structure, favored a tentative x-ray diagnosis of odontoma.

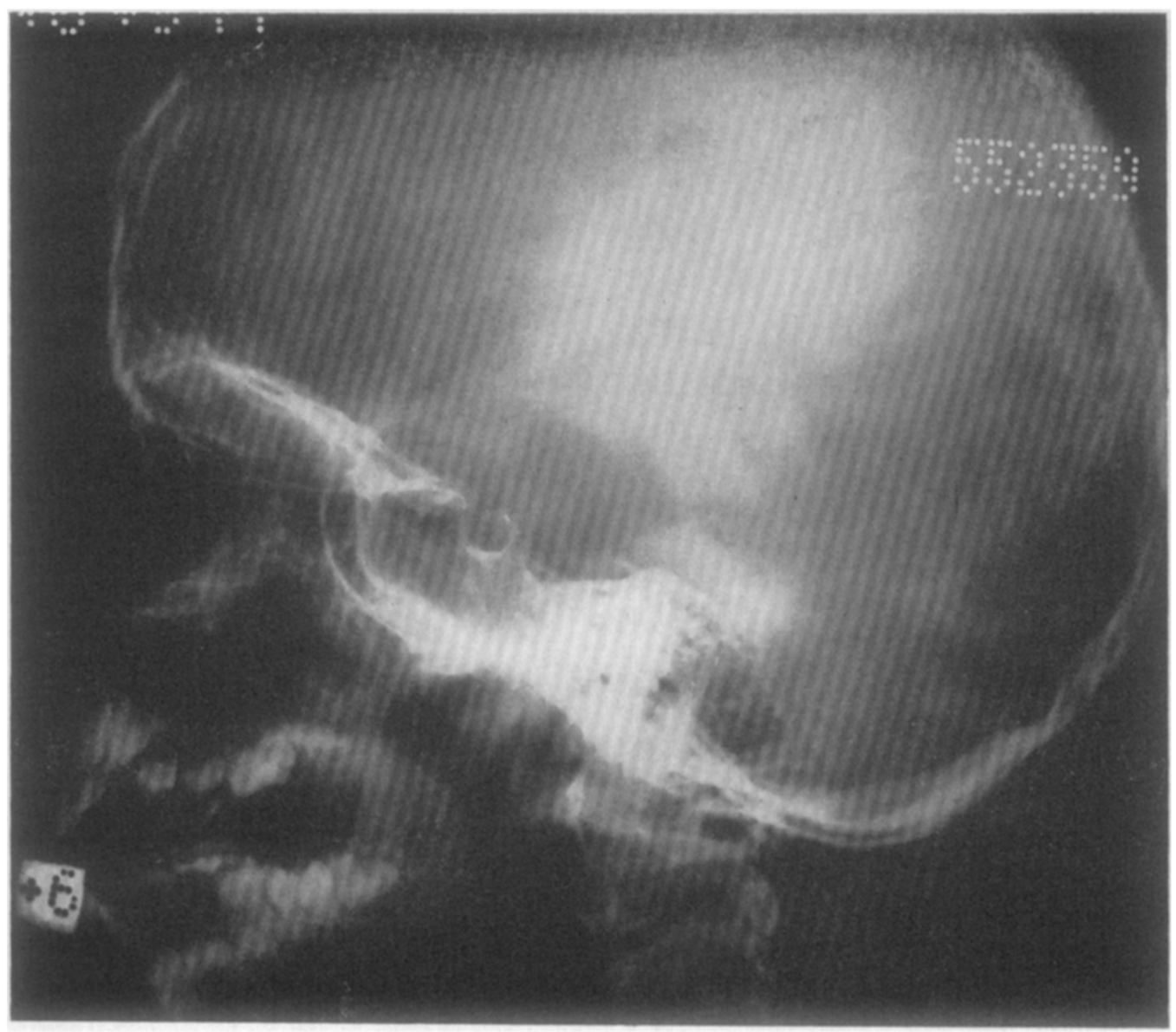

Fig. 6.-See opposite page for legend.

Because of the history of rapid growth and the loose second deciduous molar, it was felt that a microscopic evaluation to rule out sarcoma was indicated.

A biopsy was made from the crest of the alveolar ridge including the mobile molar. This showed, on microscopic examination, myxomatous connective tissue with an occasional nest of cells with a palisade suggesting adamantinoma. There was also a small eyst lined by columnar epithelium. 
The patient was operated on under intratracheal nitrous oxide and ether anesthesia. An incision was made on either side of the crest of the alveolar ridge, parallel to and about $1 \mathrm{~cm}$. above the gingival margins. These were carried from the incisor region to the tuberosity so as to include tissue well outside the biopsied area. The mucoperiosteum was elevated from the buccal and palatal processes. The buccal plate of bone was parchment paper in thickness, but otherwise it, as well as the palatal process, appeared normal.

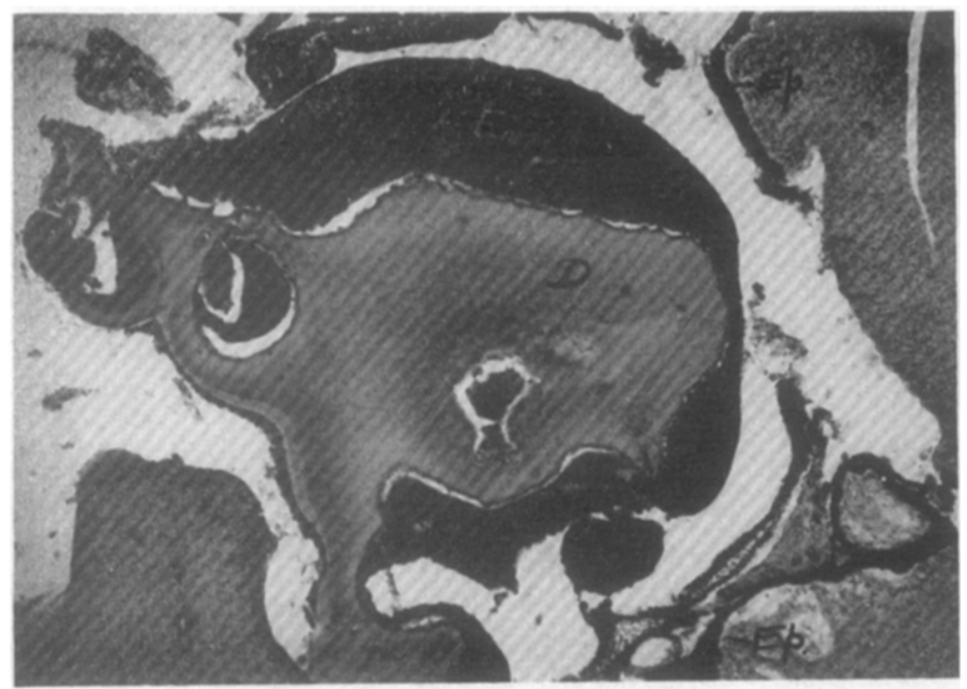

Fig. 7.- Photomicrograph showing atypical tooth structure. Irregular mass of dentine surrounded by enamel matrix. Epithelial elements on periphery of mass. En, Enamel; Ep, epithelium; $D$, dentine. Hematoxylin and eosin. $\times 40$.

A large section of the thin buccal plate was removed, exposing an encapsulated tumor which stripped away from the bony walls with ease. In order to give access to the anterior pole of the tumor, the alveolar process, including the first deciduous molar, was removed. This exposed the unerupted crowns of the two premolars which were lying in a dense cellular tissue. The neoplasm extended anteriorly to the deciduous cuspid, which also was extracted and showed erosion of the posterior portion of the upper third of its root. Beginning anteriorly, the heavy capsule was easily teased free from the walls of the bony cavity. The tumor was enucleated as far back as the posterior extremity of the maxilla, where it was found to have eroded through the zygomatic surface and tuberosity and to have extended into and completely filled the pterygopalatine fossa. By gentle and steady traction the posterior portion was completely extirpated. The capsule of the tumor was very thick and intact except for a wide rupture at the point where a section had previously been excised for a histological examination. Together with the complete neoplasm, the surgical specimen also included all of the excised alveolar ridge posterior to the lateral incisor as well as a large section of the buccal and palatal plate. The remaining cavity, which measured 6 by 6 by $6 \mathrm{~cm}$., was packed with a large petrolatum gauze dressing.

The gross specimen consisted of a tumor 6 by 6 by $6 \mathrm{~cm}$. completely covered by a very thick leathery capsule. On sectioning, it consisted of a soft, pink, 
fleshy mass, containing a very large number of calcified bodies varying in size and shape. Some resembled rudimentary teeth. There were two well-formed molar crowns (probably first and second molars), as well as two conglomerate masses measuring 1 by $2 \mathrm{~cm}$.

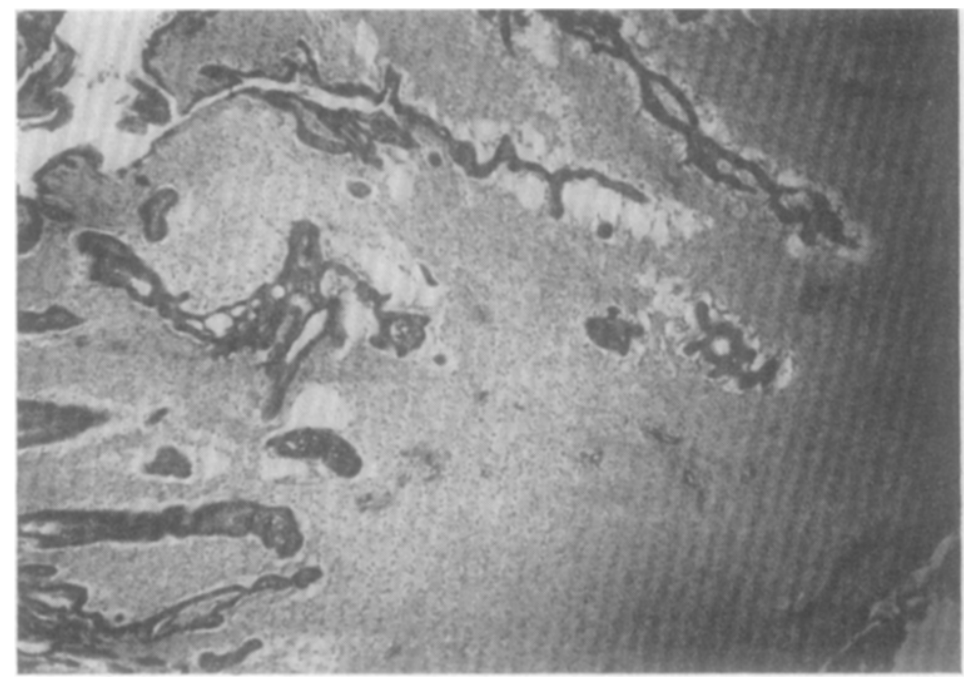

Flg. 8.-Cords and masses of epithelial cells in delicate connective tissue stroma. Typical areas of adamantinoma. Hematoxylin and eosin. $\times 40$.

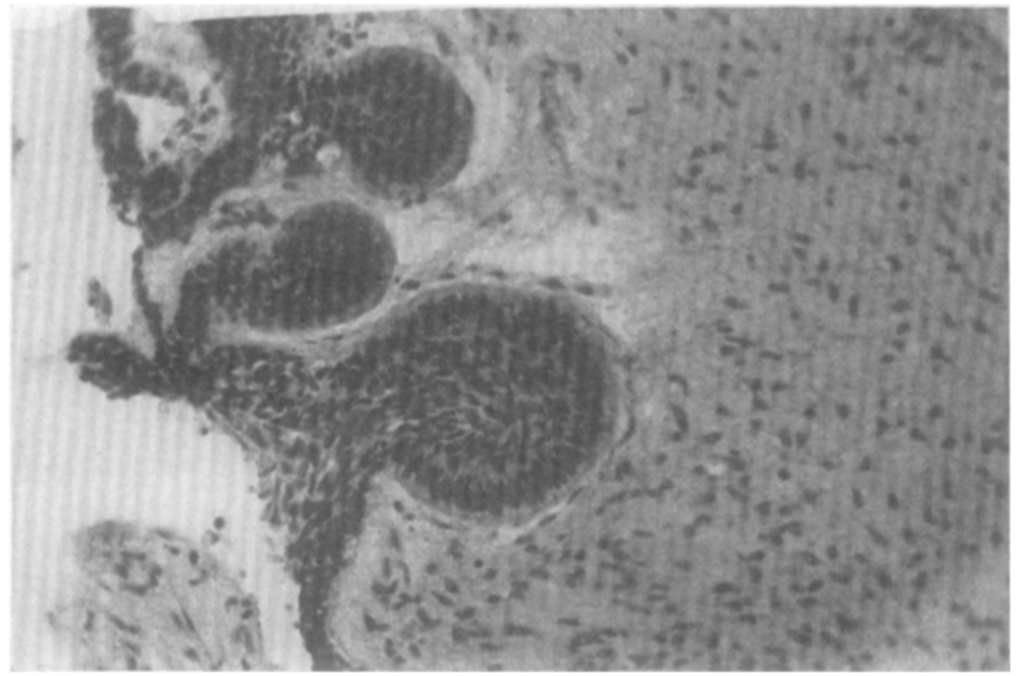

Fig. 9.-Higher magnification of epithelial mass showing characteristic morphology and arrangement of cells. Hematoxylin and eosin. $\times 100$.

Microscopic Examination.--"The specimen received measured 5 by 6 by 6 cm. and was surrounded by a dense fibrous capsule. The mass cut with diffi culty because of the numerous areas of calcification. One cut section of the interior of the mass was a conglomerate of varying sized glistening white structures, some of which appeared to be atypical teeth. In other areas the mass was more solid, had a mottled appearance, and contained areas of calcification. 
"The periphery of the mass was dense fibrous connective tissue in which high cuboidal to high columnar epithelial cells were found in masses, strands, and anastomosing cords. The cells were arranged in a parallel or palisade fashion at the margin of the masses and cords. Many cells were tall, columnar, with basally placed nuclei, and appeared to be atypical ameloblasts. Toward the interior of the mass these groups of cells became larger and surrounded small cystic spaces. They also were found associated with masses of embryonic connective tissue. The main bulk of the interior of the structure was composed of masses of enamel matrix, dentine, and cellular and acellular cementum in an atypical relationship. This therefore is a complex neoplasm showing typical areas of ameloblastoma and the characteristics of a complex composite odontoma. Diagnosis: Adamanto-odontoma." (Figs. 7 to 10.)

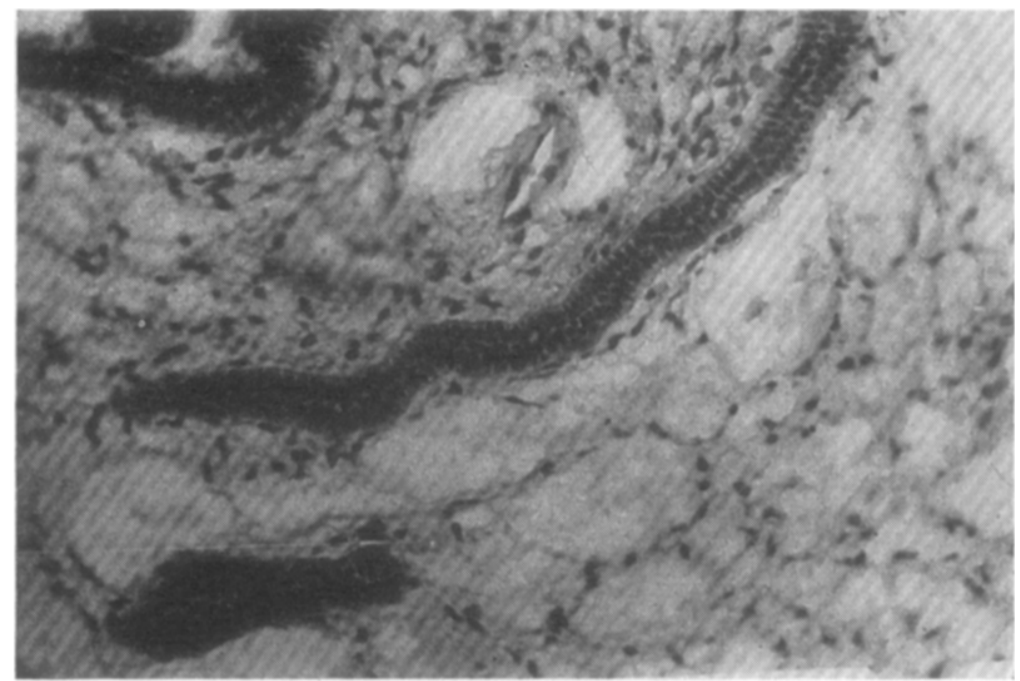

Fig. 10.-Higher magnification of cord of epithelial cells showing characteristic morphology and arrangement of cells. Hematoxylin and eosin. $\times 100$.

The operative course was uneventful. The dressings were changed every third day postoperatively, decreasing the size of the pack at each sitting. The patient was discharged to the care of her local physician with instructions to report for re-examination in three months.

Discussion.- The history of rapid growth of a noninflammatory tumor of the maxilla in a 6-year-old child, together with the marked mobility of a noncarious, vital deciduous second molar, strongly suggested that we might be dealing with a malignant neoplasm, possibly a sarcoma. After obtaining the radiographic evidence of the marked degree of expansion by the tumor of the superior and lateral walls of the maxillary sinus with no demonstrable erosion of the bone, we favored the diagnosis of a benign tumor. The numerous calcified bodies, appearing as a more or less conglomerate mass containing what appeared to be the crowns of unerupted premolars and permanent molars, favored the radiographic diagnosis of cystic odontoma. This was a possibility in this case since odontomas develop and may increase to a large size during the period of dental development. 
Radiographically there was nothing to suggest any of the other usual tumors of the jaw, and a positive evaluation by microscopic examination was indicated before surgery was instituted. With the pathologic diagnosis of adamantinoma, the question arose as to how radical should be the treatment. It is generally agreed that conservatism in the treatment of adamantinoma frequently results in recurrence and many of the cases so handled eventually require further surgery. Although adamantinoma may be considered a benign tumor, instances of metastasis have occurred. Malignant degenerative changes are apt to occur with frequent recurrences.

Yet in this case, because of the heavy capsule which afforded easy and apparently complete enucleation, and the fact that its location offered technical difficulties and surgical restrictions, radical surgery was contraindicated. It was felt that a mutilating operation should be done only in case of future recurrence.

This is a case of a rare, mixed odontogenic tumor. Associated with the development of a dental anomaly (odontoma) there was also a proliferation of the epithelial elements to create an ameloblastic tumor. 